\title{
Targeted Mass Spectrometric Immunoassay for Detection of Cystatin C Isoforms in Cerebrospinal Fluid
}

\author{
Dobrin Nedelkov $^{1, *}$, Shainag Shaik ${ }^{1}$, Olgica Trenchevska ${ }^{2}$, Vasko Aleksovski $^{3}$, Angel Mitrevski $^{3}$ \\ and Kiro Stojanoski ${ }^{2, *}$
}

${ }^{1}$ Intrinsic Bioprobes Inc., Tempe, AZ, USA; ${ }^{2}$ Institute of Chemistry, Faculty of Sciences, Sts. Cyril and Methodius University, Skopje, Macedonia and ${ }^{3}$ Faculty of Medicine, Sts. Cyril and Methodius University, Skopje, Macedonia

\begin{abstract}
Targeted cerebrospinal fluid (CSF) Mass Spectrometric Immunoassay for cystatin C was developed and applied to a small number of CSF and serum samples obtained from healthy and individuals diagnosed with multiple sclerosis. Cystatin C and its variants were retrieved from the CSF samples via affinity micro columns derivatized with cystatin $\mathrm{C}$ antibodies, and were eluted onto MALDI mass spectrometer targets. The resulting mass spectra revealed various levels of cystatin $\mathrm{C}$ isoforms in the samples. Truncated Cystatin $\mathrm{C}$ isoforms missing 3, 4, 7, 8, 9, 10, 11, 14, 15, and $17 \mathrm{~N}$ terminal amino acid residues were detected. Correlation in the level of truncation between the matched CSF and serum samples was also observed. The mass spectrometric immunoassay provided rapid, high-throughput assessment of cystatin $\mathrm{C}$ and its isoforms present in biological fluids.
\end{abstract}

Key Words: Cystatin C, CSF, mass spectrometry, immunoassay, serum.

\section{INTRODUCTION}

Sandwich-based immunoassays are the mainstay in quantitative protein analysis. However, these protein-specific immunoassays utilize detection methods and labels that cannot discriminate potential structural protein modifications due to the fact that the resulting quantitative signal is the sum of signals from all isoforms for a given protein captured by the primary affinity ligands. Because these protein isoforms can serve as indicators (biomarkers) of disease onset and progression, there is a clear need for the development of high throughput methods for their qualitative detection and quantification. Proteomics, with its enabling mass spectrometric detection is well suited for the assessment of these protein variants. Nevertheless, most MS-based proteomics methods employ fractionation approaches (e.g., 2 DE, LC, etc) that are complex, multifaceted, low-throughput, and hard to reproduce on a daily basis. Furthermore, the current emphasis in proteomics is on the "group" aspect rather than on "individual" proteins, which can lead to content-rich, highly convoluted, and quantification-challenging data sets that require powerful informatics approaches for results interpretation.

One way of overcoming these limitations of the standard immunoassays and the proteomics approaches is to combine immunoaffinity protein capture with mass spectrometric methods of detection. In this approach, a primary antibody is utilized for affinity capture of a targeted protein, while the detection is performed by mass spectrometry, which provides direct readout of the protein molecular mass. Hence, if

*Address correspondence to these authors at the 2155 E. Conference Dr. Suite 104, Tempe AZ 85284 USA; Tel: +1-480-804-1778; Fax: +1-480804-0778; dnedelkov@intrinsicbio.com and kiro@pmf.ukim.edu.mk the antibody (preferably polyclonal) captures all protein isoforms present in the sample, the ensuing MALDI TOF MS analysis will yield mass spectra in which the signals for the isoforms are clearly set apart from the signal of the wild type protein. In one form of immunoaffinity mass spectrometry termed Mass Spectrometric Immunoassay (MSIA) [1], protein(s) are extracted from the biological sample with the help of affinity pipettes derivatized with polyclonal antibodies (Fig. 1). The proteins are then eluted from the affinity pipettes with a MALDI matrix, deposited onto a target, and analyzed in a MALDI-TOF mass spectrometer. The MSIA assays have mainly been used for assaying human plasma or serum proteins, and have thus far not been applied to analysis of proteins from cerebrospinal fluid (CSF). In this work we develop and apply a high-throughput MSIA assay for analysis of cystatin C in CSF. Cystatin C is a cysteine proteinase inhibitor ubiquitous to all biological fluids, and has been indicated in a number of ailments $[2,3]$.

\section{MATERIALS AND METHODOLOGY}

Rabbit anti-human polyclonal antibody to cystatin $\mathrm{C}$ was obtained from DAKO (A0451, $17 \mathrm{~g} / \mathrm{L}$, Carpinteria, CA, USA). CDI (1,1'-Carbonyldiimidazole) - activated affinity pipettes (Intrinsic Bioprobes Inc., Tempe, AZ) were prepared as previously described [4]. To immobilize the antibody, the activated affinity pipettes were mounted onto the head of a Beckman Multimek Automated 96-Channel Pipettor (Beckman Coulter, Fullerton, CA, USA) and immediately immersed into the wells of a 96-well microplate containing 100 $\mu \mathrm{L}$ of 100 -fold diluted antibody solution (in $10 \mathrm{mM}$ sodium acetate, $\mathrm{pH}$ 5.0). The antibody was covalently coupled to the CDI-activated surface by repetitively flowing $50 \mu \mathrm{L}$ the solution over the pipettes (400 cycles, each cycle consisting of a single aspiration and dispense through the affinity pi- 


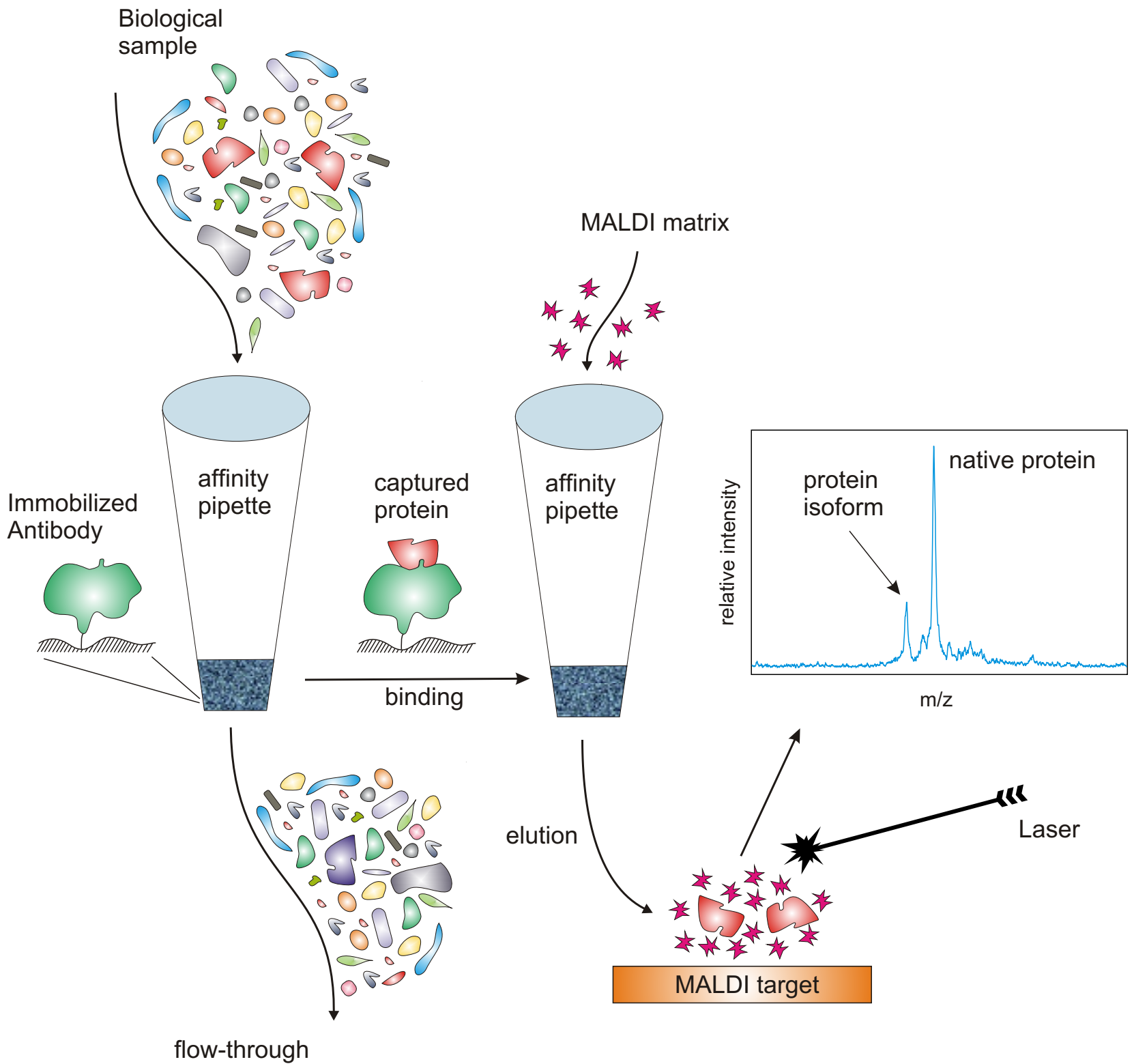

Fig. (1). Schematics of the MSIA approach.

pettes). Next, the pipettes were immersed into a microplate containing $150 \mu \mathrm{L}$ of $1 \mathrm{M}$ solution of ethanolamine in each well, and 50 aspirations and dispense cycles $(100 \mu \mathrm{L}$ volumes each) were performed, to block the un-reacted carboxyl groups. The pipettes were then rinsed twice with $60 \mathrm{mM} \mathrm{HCl}$ (10 cycles each) and twice with HBS physiological buffer (HEPES-buffered saline, $10 \mathrm{mM}$ HEPES $\mathrm{pH} 7.4,150 \mathrm{mM}$ $\mathrm{NaCl}, 2$ x 10 cycles), after which the antibody-derivatized affinity pipettes were stored at $4{ }^{\circ} \mathrm{C}$ until use.

The cerebrospinal fluid samples (12 from individuals diagnosed with multiple sclerosis and 5 healthy controls) and 6 matching serum samples (4 multiple sclerosis and 2 controls) were obtained from the Clinic of Neurology, Faculty of Medicine in Skopje, Macedonia. The diagnosis of multiple sclerosis was made following standard criteria [5, 6]. The samples were labeled only with a barcode and an accompanying specification sheet containing information about the disease state, ensuring proper privacy protection. A $15 \mu \mathrm{L}$ aliquot of each sample was placed into a particular well on a 96-well microplate and diluted with $135 \mu \mathrm{L}$ of HBS buffer to a 10-fold working sample dilution. The antibody-derivatized affinity pipettes were mounted onto the head of the Multimek pipettor and initially rinsed with $100 \mu \mathrm{L}$ of HBS buffer (10 cycles). Next, the pipettes were immersed into the sample tray and 150 aspirations and dispense cycles $(100 \mu \mathrm{L}$ volumes each) were performed, allowing for affinity capture of the targeted protein. The pipettes were then rinsed with HBS (10 cycles), water (10 cycles), $2 \mathrm{M}$ ammonium acetate - acetonitrile $(3: 1 \mathrm{v} / \mathrm{v})$ mixture (10 cycles), and two final water rinses (10 cycles each). The affinity pipettes containing the retrieved proteins were then rinsed with $1 \mathrm{mM} \mathrm{N}$ octylglucoside (single cycle with a $150 \mu \mathrm{L}$ aliquot) in order to homogenize the subsequent MALDI matrix draw and elution by completely wetting the porous affinity supports inside the pipettes. For elution of the captured proteins, $6 \mu \mathrm{L}$ aliquots of MALDI matrix (6 g/L $\alpha$-cyano-4-hydroxycinnamic acid in aqueous solution containing $33 \%(\mathrm{v} / \mathrm{v})$ acetonitrile, and $0.4 \%(\mathrm{v} / \mathrm{v})$ trifluoroacetic acid) were aspired 
into the affinity pipettes, and after a 10 second delay (to allow for the dissociation of the protein from the capturing antibody), the eluates containing the targeted protein were dispensed directly onto a 96-well formatted MALDI target. Linear mass spectra were acquired on an Autoflex MALDITOF mass spectrometer (Bruker Daltonics, Billerica, MA), with a delayed extraction using a $1.8 \mathrm{kV}$ draw out pulse, 200 $\mathrm{ns}$ delay, and a full accelerating potential of $20.00 \mathrm{kV}$. Two hundred laser shots were acquired for each spectrum. The resulting mass spectra were evaluated using the Zebra software (Intrinsic Bioprobes, Tempe, AZ), which, when used in conjunction with PAWS (sequence display and manipulation software from Proteometrics, NY), allows for rapid identification of protein sequence modifications via display of mass values and the mass differences between peaks. A peak was assigned to a specific modification if the observed $\mathrm{m} / \mathrm{z}$ value differed by less than $0.02 \%$ from that empirically predicted. The MSIA assays were performed in triplicate.

\section{RESULTS AND DISCUSSION}

A small number of CSF (and few matched serum) samples were analyzed to assess the ability of the newly developed CSF cystatin C MSIA assay to rapidly detect and quantify cystatin $C$ truncated isoforms. Cystatin $C$ have been analyzed with MSIA in the past, both from human plasma [7] and urine samples [8], but not from CSF samples. The analysis of CSF entailed several assay optimization steps, the most important being the dilution factor for the samples, the dilution buffer, and the incubation time of the affinity pipettes with the samples. It was determined empirically that best results were obtained when CSF was diluted 10-fold into HEPES-buffered saline. Affinity capture was achieved during 150 aspiration and dispense cycles. The rinsing protocols and matrix elution/deposition steps remained identical to those previously reported for plasma [7].

Shown in Figs. (2 and 3) are representative mass spectra for each sample resulting from the triplicate MSIA analyses of the 12 multiple sclerosis (\#1-12) and 5 healthy controls (\#13-17) CSF samples, and 6 matched serum samples (\#1, 3, $8,9,13$, and 16; the numbers correspond to those for the CSF samples), respectively. The signals observed are all in the $\mathrm{m} / \mathrm{z}$ region of the spectra where native cys $\mathrm{C}$ and its truncated isoforms should register. Notable is the absence of peaks due to other non-specifically retained proteins, with the exception of a peak at $\mathrm{m} / \mathrm{z}$ of 12,120 (labeled with a *), which is an artifact of the antibody coupling process (this peak is observed in almost all of the MSIA analyses when DAKO-made antibodies are immobilized in the affinity pipettes). Native CysC (MW=13,343) was observed in all but 3 of the mass spectra. The rest of the peaks represent cystatin $\mathrm{C}$ isoforms that lack several $\mathrm{N}$-terminal amino acids. More specifically, truncated Cystatin $\mathrm{C}$ isoforms missing 3, 4, 7, 8, $9,10,11,14,15$, and $17 \mathrm{~N}$-terminal amino acid residues were observed. The abundance of the isoforms, as evidenced by the height of the signals compared to the native $\mathrm{CysC}$ peak, varied significantly from sample to sample. In some samples, such as \#6, 9 and 17, they were barely observed. In others, such as \#1,2, and 11, they represent the dominant cystatin $\mathrm{C}$ signals in the mass spectra. This wide range of cystatin $\mathrm{C}$ isoforms abundance in CSF has not been reported before. In a recent study of 1,000 human plasma samples, we have catalogued the presence of a number of cystatin $\mathrm{C}$ iso- forms, more specifically those lacking $1,3,4,5,7,8,10$, and $11 \mathrm{~N}$-terminal amino acids $[9,10]$. Most abundant were the short-cleaved isoforms (those missing 4 or less $\mathrm{N}$-terminal amino acids): des-S, des-SSP, and des-SSPG, observed in $30 \%$ or more of the 1,000 samples. The des-SSPGKPPR was most abundant from the long-cleaved isoforms, and it was observed in $3 \%$ of the 1,000 plasma samples. In the present study, this 8-amino acid truncated cystatin $\mathrm{C}$ isoform was observed in 11 of the 17 CSF samples, and in 5 of the 6 serum samples, which is at a higher percentage than the plasma samples study. Most importantly, the isoforms lacking 14, 15 , and 17 amino acids reported here were not detected in the 1,000 plasma samples.

The presence of truncated cystatin $\mathrm{C}$ isoforms in CSF samples obtained from patients suffering from multiple sclerosis has been the subject of a number of recent investigations. Irani et al. reported a correlation among the ratio of the des-SSPGKPPR cysC and native CysC peaks, and the occurrence of multiple sclerosis [11]. However, several other groups have studied this $12.5 \mathrm{kDa}$ cleavage product of Cystatin $\mathrm{C}$ and had came up with a conclusion that the degradation was a result of prolonged storage at $-20 \mathrm{C}$, and not specific to multiple sclerosis [12-14]. The samples analyzed in this study were obtained during a 7 months period beginning in May of 2006 and were stored initially at $-20{ }^{\circ} \mathrm{C}$ for couple of months, followed by $-70{ }^{\circ} \mathrm{C}$ storage upon their delivery to the analytical laboratory where the MSIA analyses were performed. Hence, it is quite possible that some cystatin truncations were storage-related. Consequently, we cannot engage in meaningful comparison of the isoforms and their abundance across the two cohorts (moreover, the number of samples analyzed in this study was relatively small). However, the MSIA assays designed here and the preliminary results obtained with their application on CSF samples clearly indicate the ability to determine such correlations in a larger study of well characterized cohorts.

Analysis of the results obtained from the matched CSF and serum samples (4 from multiple sclerosis patients, 2 from healthy controls) reveals some interesting correlations. For example, serum sample \#1 showed most extensive truncations, similar to CSF sample \#1, both of which were obtained from the same individual. Similar trends in the truncation patterns were observed for the other serum-CSF matched samples, with exception of sample \#13 for which the serum showed higher amounts of truncated cystatin $\mathrm{C}$ isoforms. From a diagnostic point of view, it is of great advantage to analyze the same biomarkers of disease in more than one biological fluid from the same individual. Albeit on a small scale, the results shown here indicate that such studies are possible and straightforward if the right assays are utilized. The targeted mass spectrometric immunoassay approach provides a unique way of delineating protein isoforms and their abundance in biological fluids, enabling large scale population proteomics studies that will provide further insight into the physiology of biological processes and diseases.

\section{ACKNOWLEDGMENTS}

The work reported in this publication was supported in part by Grant No. 094505 from the Ministry of Education and Science of the Republic of Macedonia. 


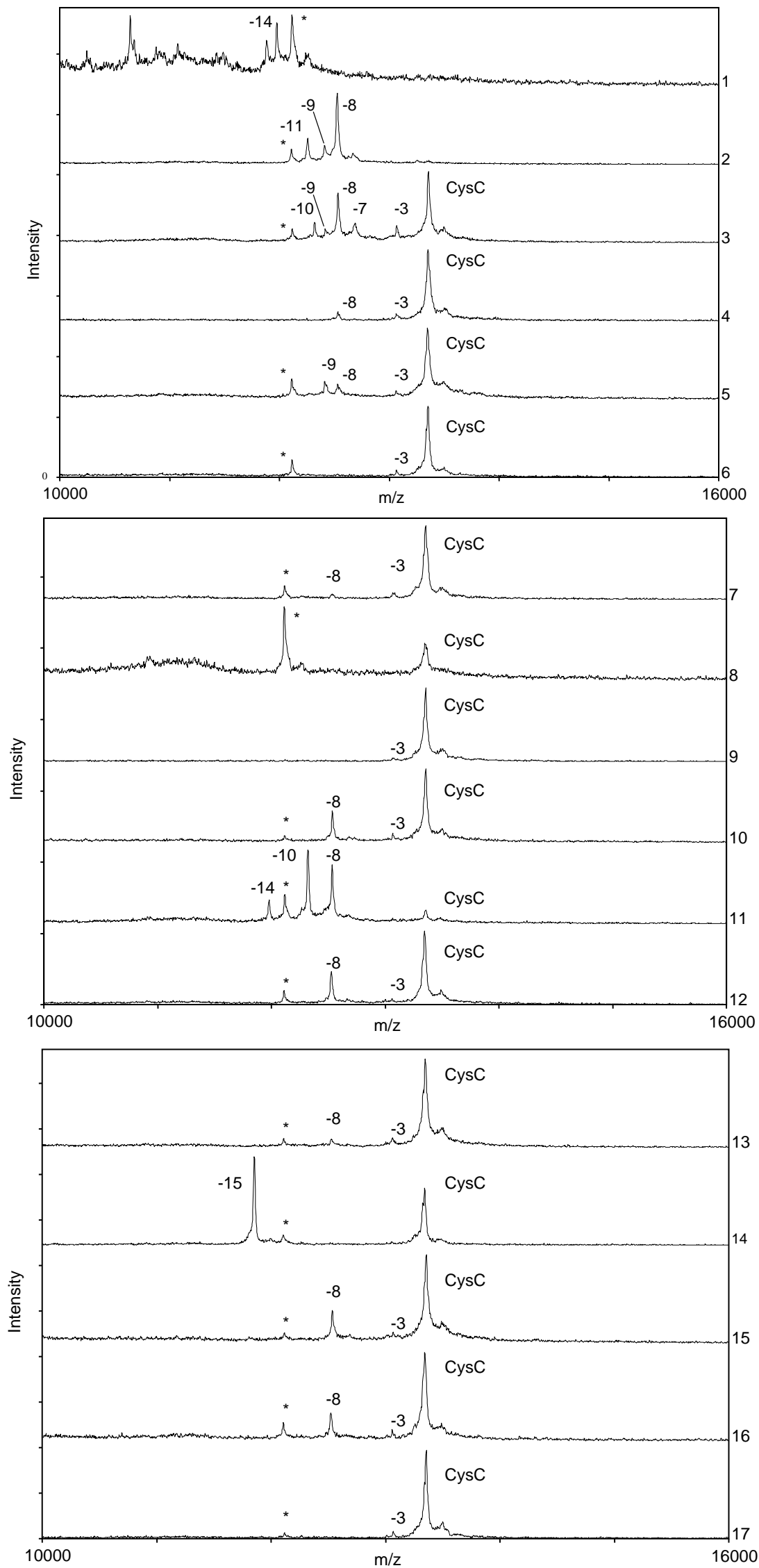

Fig. (2. Mass spectra resulting from the MSIA analysis of 12 multiple sclerosis-diagnosed (samples \#1-12) and 5 healthy controls CSF samples (13-17). The peaks are labeled as native cystatin $\mathrm{C}$ (CysC), isoforms missing specific number of $\mathrm{N}$-terminal amino acids (-3, -4 , etc), and artifacts from the antibody immobilization $(*)$. The numbers at the right side of the spectra denote the sample number. 


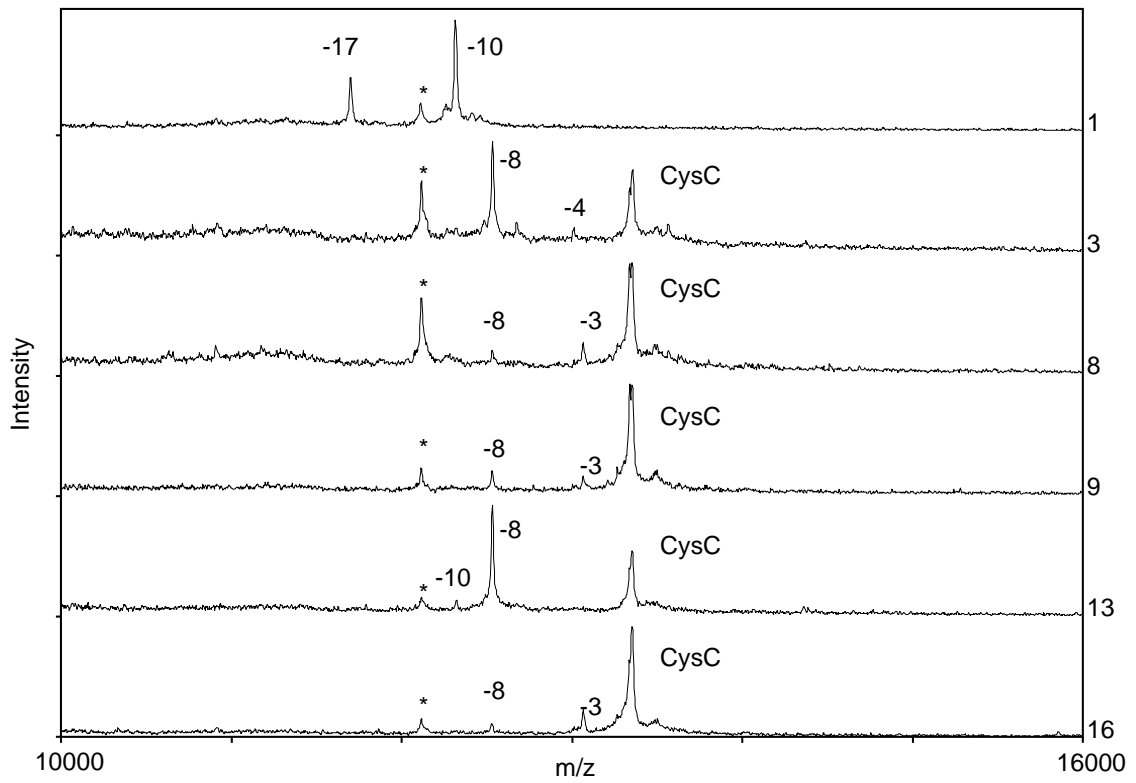

Fig. (3). Mass spectra resulting from the MSIA analysis of 6 matched serum samples (samples \#1, 3, 8, 9, 13, and 16). The peak labeling is as in Fig. (2).

\section{REFERENCES}

[1] Nedelkov, D. Expert Rev. Proteom., 2006, 3, 631-640.

[2] Henskens, Y. M.; Veerman, E. C.; Nieuw Amerongen, A. V. Biol. Chem. Hoppe Seyler, 1996, 377, 71-86.

[3] Grubb, A. O. Adv. Clin. Chem., 2000, 35, 63-99.

[4] Niederkofler, E. E.; Tubbs, K. A.; Kiernan, U. A.; Nedelkov, D.; Nelson, R. W. J. Lipid Res., 2003, 44, 630-639.

[5] McDonald, W. I.; Compston, A.; Edan, G.; Goodkin, D.; Hartung, H. P.; Lublin, F. D.; McFarland, H. F.; Paty, D. W.; Polman, C. H.; Reingold, S. C.; Sandberg-Wollheim, M.; Sibley, W.; Thompson, A.; van den Noort, S.; Weinshenker, B. Y.; Wolinsky, J. S. Ann. Neurol., 2001, 50, 121-127.

[6] Mitrevski, A.; Stojanoski, K.; Korneti, P. Acta Pharm., 2001, 51, 163-171.

[7] Kiernan, U. A.; Nedelkov, D.; Niederkofler, E. E.; Tubbs, K. A.; Nelson, R. W. Methods Mol. Biol., 2006, 328, 141-150.

[8] Kiernan, U. A.; Tubbs, K. A.; Nedelkov, D.; Niederkofler, E. E.; McConnell, E.; Nelson, R. W. J. Proteome. Res., 2003, 2, 191-197.
[9] Nedelkov, D.; Kiernan, U. A.; Niederkofler, E. E.; Tubbs, K. A.; Nelson, R. W. Proc. Natl. Acad. Sci. U.S.A., 2005, 102, 1085210857.

[10] Nedelkov, D.; Phillips, D. A.; Tubbs, K. A.; Nelson, R. W. Mol. Cell. Proteom., 2007, 6, 1183-1187.

[11] Irani, D. N.; Anderson, C.; Gundry, R.; Cotter, R.; Moore, S.; Kerr, D. A.; McArthur, J. C.; Sacktor, N.; Pardo, C. A.; Jones, M.; Calabresi, P. A.; Nath, A. Ann. Neurol., 2006, 59, 237-247.

[12] Nakashima, I.; Fujinoki, M.; Fujihara, K.; Kawamura, T.; Nishimura, T.; Nakamura, M.; Itoyama, Y. Ann. Neurol., 2006, 62, 197-200.

[13] Hansson, S. F.; Hviid Simonsen, A.; Zetterberg, H.; Andersen, O.; Haghighi, S.; Fagerberg, I.; Andreasson, U.; Westman-Brinkmalm, A.; Wallin, A.; Ruetschi, U.; Blennow, K. Ann. Neurol., 2006, 62, 193-196.

[14] Del Boccio, P.; Pieragostino, D.; Lugaresi, A.; Di Ioia, M.; Pavone, B.; Travaglini, D.; D'Aguanno, S.; Bernardini, S.; Sacchetta, P.; Federici, G.; Di Ilio, C.; Gambi, D.; Urbani, A. Ann. Neurol., 2006, 62, 201-204.

(C) Nedelkov et al.; Licensee Bentham Open.

This is an open access article distributed under the terms of the Creative Commons Attribution License (http://creativecommons.org/licenses/by/2.5/), which permits unrestrictive use, distribution, and reproduction in any medium, provided the original work is properly cited. 